\title{
THE
}

\section{Partitioning of perfluorooctanoate into phosphatidylcholine bilayers is chain length-independent}

Wei Xie

Geoffrey D. Bothun

University of Rhode Island, gbothun@uri.edu

Hans-Joachim Lehmler

Follow this and additional works at: https://digitalcommons.uri.edu/che_facpubs

Part of the Chemical Engineering Commons

This is a pre-publication author manuscript of the final, published article.

Creative Commons License

(c) (i) (8)

This work is licensed under a Creative Commons Attribution-Noncommercial-No Derivative Works 4.0 License.

\section{Citation/Publisher Attribution}

Xie, W., Bothun, G. D., \& Lehmler, H.-J. (2010). Partitioning of perfluorooctanoate into phosphatidylcholine bilayers is chain length-independent. Chemistry and Physics of Lipids, 163(3), 300-308. doi: 10.1016/ j.chemphyslip.2010.01.003

Available at: https://doi.org/10.1016/j.chemphyslip.2010.01.003

This Article is brought to you for free and open access by the Chemical Engineering at DigitalCommons@URI. It has been accepted for inclusion in Chemical Engineering Faculty Publications by an authorized administrator of DigitalCommons@URI. For more information, please contact digitalcommons-group@uri.edu. 


\title{
PARTITIONING OF PERFLUOROOCTANOATE INTO PHOSPHATIDYLCHOLINE BILAYERS IS CHAIN LENGTH- INDEPENDENT
}

\author{
Wei Xie ${ }^{a}$, Geoffrey D. Bothun ${ }^{b}$, and Hans-Joachim Lehmler ${ }^{a}{ }^{*}$ \\ ${ }^{a}$ Department of Occupational and Environmental Health, University of lowa, College of Public \\ Health, lowa City, IA 52242, USA

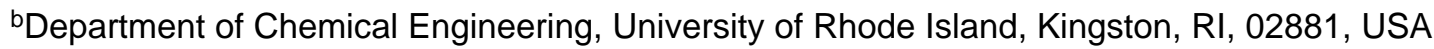

\begin{abstract}
The chain length dependence of the interaction of PFOA, a persistent environmental contaminant, with dimyristoyl- (DMPC), dipalmitoyl- (DPPC) and distearoylphosphatidylcholine (DSPC) was investigated using steady-state fluorescence anisotropy spectroscopy, differential scanning calorimetry (DSC) and dynamic light scattering (DLS). PFOA caused a linear depression of the main phase transition temperature $T_{m}$ while increasing the width of the phase transition of all three phosphatidylcholines. Although PFOA's effect on the on $T_{m}$ and the transition width decreased in the order DMPC > DPPC > DSPC, its relative effect on the phase behavior was largely independent of the phosphatidylcholine. PFOA caused swelling of DMPC but not DPPC and DSPC liposomes at $37^{\circ} \mathrm{C}$ in the DLS experiments, which suggests that PFOA partitions more readily into bilayers in the fluid phase. These findings suggest that PFOA's effect on the phase behavior of phosphatidylcholines depends on the cooperativity and state (i.e., gel versus liquid phase) of the membrane. DLS experiments are also consistent with partial liposome solubilization at PFOA/lipid molar ratios $>1$, which suggests the formation of mixed PFOA-lipid micelles.
\end{abstract}

\section{Keywords}

Fluorinated surfactants; perfluorooctanoate; model membranes; partition coefficients

\section{Introduction}

Perfluorinated surfactants, such as perfluorooctanoate (PFOA), have been used for over 50 years in various large scale technical applications such as fire extinguishing media and electroplating baths, the preparation of fluoropolymers, as well as in consumer products such as stain-repellent coatings on carpets, paper or fabrics, lubricants, and personal care and cleaning products (Kissa, 2001; Lehmler, 2005). The multifaceted use of perfluorinated surfactants results from an unusual combination of properties such as thermal, chemical and

\section{(C) 2010 Elsevier Ireland Ltd. All rights reserved.}

*Corresponding Author: Dr. H.-J. Lehmler, The University of Iowa, Department of Occupational and Environmental Health, 100 Oakdale Campus \#221 IREH, Iowa City, IA 52242-5000, Phone: (319) 335-4414, Fax: (319) 335-4290, hans-joachim-lehmler@uiowa.edu.

Publisher's Disclaimer: This is a PDF file of an unedited manuscript that has been accepted for publication. As a service to our customers we are providing this early version of the manuscript. The manuscript will undergo copyediting, typesetting, and review of the resulting proof before it is published in its final citable form. Please note that during the production process errors may be discovered which could affect the content, and all legal disclaimers that apply to the journal pertain. 
biological inertness, excellent spreading characteristics, and high surface activity. In particular, perfluorooctanoic acid and homologous perfluorinated acids have emerged as compounds of environmental concern and have been detected in various environmental matrices and in humans (Lau et al., 2007). Their extreme inertness towards chemical and biological degradation as well as their hydrophobic character contribute to their persistence in the environment and the accumulation of medium-to-long chain perfluorinated surfactants in the food chain (Martin et al., 2003a, b). This worldwide contamination with perfluorinated surfactants gives rise to environmental as well as human health concerns.

Like other perfluorinated environmental contaminants (Lau et al., 2007), in utero exposure to PFOA has been linked to significant neonatal mortality in mice (Lau et al., 2006). Although peroxisome proliferator activated receptor alpha $(\operatorname{PPAR} \alpha)$ appears to play a crucial role in the developmental toxicity of PFOA (Abbott et al., 2007; Rosen et al., 2007), non-PPAR $\alpha$ modes of action may play an important role as well (Lau et al., 2007; Rosen et al., 2007). Specifically, the inhibition of pulmonary surfactant has been proposed as one mechanism that may cause or contribute to the neonatal mortality in mice (Xie et al., 2007). This "respiratory distress" hypothesis is based on the observation that perfluorinated environmental contaminants, such as PFOA and perfluorooctanesulfonic acid (PFOS), not only partition readily into lipid assemblies, but also have a significant effect on their phase transition (Hu et al., 2003; Inoue et al., 1988a; Matyszewska et al., 2007; Xie et al., 2007). Furthermore, monolayer studies at the air-water interface show that PFOA and related perfluoroalkyl acids partition into the lipid monolayer (Arora et al., 2003; Lehmler and Bummer, 2004; Matyszewska et al., 2007; Nakahara et al., 2005) and alter the dynamic function of pulmonary surfactant in vitro (Gordon et al., 2007). In particular, monolayer studies suggest that PFOA-phospholipid interactions may depend on the fluidity and, possibly, the cooperativity of the phase transition of the lipid assembly.

Unfortunately, systematic studies investigating the role of the cooperativity of the main phase transition in PFOA-phospholipid interactions have not been reported. To close this gap in knowledge, the present study investigates the interaction of PFOA with model membranes prepared from symmetrical phospholipids with different tail lengths using fluorescence anisotropy measurements, differential scanning calorimetry (DSC) and dynamic light scattering (DLS). Phosphatidylcholine liposomes were chosen for these studies because they are the primary phospholipids present in pulmonary surfactant (Goerke, 1998; Veldhuizena et al., 1998).

\section{Materials and methods}

\subsection{Chemicals}

Potassium perfluorooctanoate $\left(\mathrm{CF}_{3}\left(\mathrm{CF}_{2}\right)_{6} \mathrm{CO}_{2} \mathrm{~K}\right)$ was prepared by neutralization of perfluorooctanoic acid with potassium hydroxide. Its analytical data are in agreement with the proposed structure. Dimyristoylphosphatidylcholine (DMPC), dipalmitoylphosphatidylcholine (DPPC) and distearoylphosphatidylcholine (DSPC) were obtained from Avanti Polar Lipids (Alabaster, AL) in $>99 \%$ purity and used without further purification. The fluorescence probes, 1,6-diphenyl-1,3,5-hexatriene (DPH) and 1-(4trimethylammoniumphenyl)-6-phenyl-1,3,5-hexatriene p-toluenesulfonate (TMA-DPH), were obtained from Molecular Probes (Eugene, OR). Tetrahydrofuran (THF), ethanol, methanol and chloroform were purchased from Fisher Scientific (Pittsburgh, PA) and were either HPLC or ACS grade. All chemicals were used without further purification. Deionized water for the DSC studies was distilled first from basic potassium permanganate followed by distillation from sulfuric acid, whereas deionized ultra-filtered water for the fluorescence and light scattering studies was obtained using Millipore water systems (Milli-Q and Direct-3Q, 
respectively, Millipore, Billerica, MA) (Lehmler and Bummer, 2004; Lehmler et al., 2006; Xie et al., 2007).

\subsection{Preparation of DPH and TMA-DPH-labeled Liposomes}

One $\mathrm{mL}$ of a solution of the respective phospholipid ( $1 \mathrm{mmol} / \mathrm{L}$ in chloroform) and $2 \mu \mathrm{L}$ of 1 $\mathrm{mmol} / \mathrm{L} \mathrm{DPH} \mathrm{(1} \mathrm{mmol/L} \mathrm{in} \mathrm{THF)} \mathrm{solution} \mathrm{were} \mathrm{mixed} \mathrm{to} \mathrm{give} \mathrm{a} \mathrm{lipid-to-probe} \mathrm{mole} \mathrm{ratio} \mathrm{of}$ 500:1. The solvents were evaporated under reduced pressure using a rotary evaporator and the resulting lipid films were dried under vacuum $(\sim 210 \mathrm{kPa})$ for at least 3 hours to remove solvent traces. The phospholipid-DPH films were hydrated with $1 \mathrm{~mL}$ ultra-pure water for 1 hour above the melting temperature of the respective phospholipid. Suspensions containing primarily large unilamellar vesicles (LUVs) were obtained by extruding the multilamellar vesicle (MLV) suspensions $\sim 15$ times through a double-stacked polycarbonate membrane filter (pore size: $200 \mathrm{~nm}$ ) using a LiposoFast extruder (Avestin Inc., British Columbia, Canada) above the melting temperature of the respective phospholipid (Bernsdorff et al., 1997).

TMA-DPH-labeled lipid films were obtained as described above. Briefly, an unlabeled phospholipid film was prepared from $1 \mathrm{~mL}$ of the respective phospholipid solution $(1 \mathrm{mmol} /$ $\mathrm{L}$ in chloroform). The phospholipid film was hydrated and labeled by mixing $1 \mathrm{~mL}$ of ultrapure water and $2 \mu \mathrm{L}$ of TMA-DPH solution ( $1 \mathrm{mmol} / \mathrm{L}$ in ethanol) and heating the mixture above this melting temperature of the respective phospholipid for 1 hour (Zhang et al., 2004). LUV suspensions were obtained from the MLV suspensions as described above for the DPHlabeled LUVs.

\subsection{Fluorescence anisotropy measurements}

Phosphatidylcholine suspensions with the desired PFOA concentrations were prepared by diluting the respective LUV suspensions 100-fold with an aqueous PFOA solution. The final PFOA concentrations ranged from 0 to $442 \mu \mathrm{mol} / \mathrm{L}$ (0 to $200 \mathrm{mg} / \mathrm{L}$ ), which corresponds to PFOA/lipid mole ratios from 0 to 44.2. DPH and TMA-DPH anisotropy in the lipids bilayer were measured using a LS55 Luminescence Spectrometer from PerkinElmer (Shelton, CT). The spectroscopic cell was temperature controlled using a PerkinElmer PTP-1 Peltier System (Shelton, CT) and was mixed with a magnetic stirrer at low speed (Lehmler et al., 2006; Xie et al., 2007). Steady-state DPH and TMA-DPH anisotropy were determined at $\lambda_{\mathrm{ex}}=350 \mathrm{~nm}$ and $\lambda_{\text {em }}=452 \mathrm{~nm}$ (Bothun et al., 2005; Lehmler et al., 2006; Xie et al., 2007), and $\lambda_{\mathrm{ex}}=360$ $\mathrm{nm}$ and $\lambda_{\mathrm{em}}=430 \mathrm{~nm}$ (Hashizaki et al., 2005; Lehmler et al., 2006; Xie et al., 2007), respectively.

The DMPC, DPPC or DSPC suspensions were equilibrated at $40^{\circ} \mathrm{C}$ (DMPC), $52.5^{\circ} \mathrm{C}$ (DPPC) or $67.5^{\circ} \mathrm{C}$ (DSPC) for 15 minutes and the DPH and TMA-DPH anisotropy values were recorded while cooling the sample to $10^{\circ} \mathrm{C}$ (DMPC), $22.5^{\circ} \mathrm{C}$ (DPPC) or $37.5^{\circ} \mathrm{C}$ (DSPC) at a rate of $0.2^{\circ} \mathrm{C} / \mathrm{min}$ (Lehmler et al., 2006; Xie et al., 2007). The temperature of the main fluidgel phase transition $\left(T_{m}\right)$, the transition width $\left(\Delta T_{r}\right)$ and the onset and completion of the transition were determined from plots of absolute fluorescence anisotropy $(\langle r\rangle)$ as a function of temperature using Sigma Plot 2002 (Version 8.0, SPSS Inc., Chicago, IL) (Inoue et al., 1988b).

\subsection{Calculation of partition coefficients}

The apparent mole fraction partition coefficient $K_{X}$ of PFOA between the lipid bilayers and the bulk aqueous phase was estimated using the equation (Inoue et al., 1988a):

$$
-\Delta T_{m}=\frac{R T_{m, o}^{2}}{\Delta H_{m}}\left(\frac{C_{S} K_{X}}{55.5+C_{L} K_{X}}\right)
$$


where $\Delta T_{m}$ is the change in melting temperature, $R$ is the gas constant, $T_{m, o}(\mathrm{~K})$ is the melting temperature of hydrated lipids, $\Delta H_{m}(\mathrm{~kJ} / \mathrm{mol})$ is the phase transition enthalpy of the respective phosphatidylcholine (Koynova and Caffrey, 1998), $C_{S}(\mathrm{M})$ is the initial aqueous concentration of PFOA, and $C_{L}(\mathrm{M})$ is the lipid concentration. In equation 1 it is assumed that (i) the phase transition enthalpy is constant and does not change with PFOA partitioning and that (ii) PFOA mixes ideally with the fluid phase and does not partition significantly into the gel phase. This method of calculating apparent partition coefficients is routinely used to determine surfactant partitioning into lipid bilayers (Inoue et al., 1988a; Inoue et al., 1986; Lehmler et al., 2006;

Xie et al., 2007).

\subsection{Differential scanning calorimetry (DSC)}

Calculated amounts of the respective phospholipids and PFOA were dissolved in chloroform : methanol $(3: 1, \mathrm{vlv})$ at the appropriate mole fractions. The PFOA/lipid mole ratios were 0 to 0.42 (DMPC and DPPC) and 0 to 0.71 (DSPC), which is approximately two and three orders of magnitude smaller than the ratios used in the fluorescence anisotropy and DLS (described below) experiments, respectively. The solvent was removed under a stream of nitrogen, the mixtures were further dried under vacuum for $\geq 3$ hours, and the samples were hydrated in an excess of water $3: 1$ by weight (Eliasz et al., 1976; Koynova et al., 1987). Samples were heated above the lipid transition temperature for 5 minutes and vortexed for 2 minutes. This process was repeated approximately eight times. Samples were stored at $4{ }^{\circ} \mathrm{C}$ for $12-16$ hours prior to DSC analysis.

A Thermal Analysis 2920 differential scanning instrument was used for the DSC studies. The hydrated samples were weighed into DSC aluminum pans. The DSC cell was purged with 60 $\mathrm{mL} / \mathrm{min}$ and the refrigerated cooling system with $120 \mathrm{~mL} / \mathrm{min}$ dry nitrogen, respectively. Samples were cooled to $4{ }^{\circ} \mathrm{C}$ at a cooling rate of $10{ }^{\circ} \mathrm{C} / \mathrm{min}$ and then heated from $4{ }^{\circ} \mathrm{C}$ to $80^{\circ}$ $\mathrm{C}$ with a heating rate of $5^{\circ} \mathrm{C} / \mathrm{min}$. The high heating rate was chosen to allow a direct comparison with previous investigations of similar systems (Eliasz et al., 1976; Koynova et al., 1987). All samples were subjected to two subsequent heating cycles. Subsequently, the onset, maximum and completion temperatures as well as peak width of the pre-transition and the main phase transition were determined for the second run using the Universal Analysis NT software (Lehmler et al., 2006; Xie et al., 2007).

\subsection{Dynamic light scattering (DLS)}

DMPC, DPPC, and DSPC samples were prepared according to the procedure described for the fluorescence anisotropy experiments. The total lipid concentration was $0.1 \mathrm{mmol} / \mathrm{L}$ in an aqueous phase consisting of deionized water and PFOA concentrations of 0 to $442 \mu \mathrm{mol} / \mathrm{L}$, which corresponded to PFOA/lipid mole ratios of 0 to 4.42 , respectively. Once prepared, the samples were placed in a $37^{\circ} \mathrm{C}$ water bath for 24 hours.

DLS measurements were conducted at $37{ }^{\circ} \mathrm{C}$ using a Brookhaven BI-200SM Goniometer equipped with a Lexel 95-2 argon laser and a BI-9000AT Digital Correlator. The photomultiplier captured scattered light at $514 \mathrm{~nm}$ through a $400 \mu \mathrm{m}$ aperture. The scattering signal was analyzed using the method of cumulants based on the $2^{\text {nd }}$ fit of the autocorrelation function to yield an average hydrodynamic radius of the samples. For a set of samples, the laser power was held constant to compare changes in scattering intensity. The root mean square of the autocorrelation function fit was less than $5 \times 10^{-3}$ for all measurements. Each sample was analyzed in duplicate and the average of these runs and standard deviation are reported. 


\section{Results}

\subsection{DPH and TMA-DPH steady-state fluorescence anisotropy}

Two fluorescent probes, DPH and TMA-DPH, were employed to investigate the effect of PFOA on the phase behavior of DMPC, DPPC and DSPC model membranes (Figure 1). In particular the TMA-DPH fluorescence anisotropy versus concentration curves showed a decrease in the anisotropy value in the gel phase of DMPC, whereas a slight increase in the anisotropy value was observed in the liquid crystalline phase of DMPC (Figure 1D). This effect of PFOA on the membrane fluidity was noted for all three phosphatidylcholines with both fluorescent probes and was more pronounced in the TMADPH studies. Furthermore, the partial phase diagram obtained for the DPH fluorescence anisotropy experiments revealed pronounced effects of increasing PFOA concentrations on the phase transition temperature $T_{m}$, the onset and completion temperatures, and the transition width of the PFOA-phosphatidylcholine mixtures (Figure 2).

A decrease of $T_{m}$ was observed with increasing PFOA concentrations for all three PFOAphosphatidylcholine mixtures (Figure 2). The apparent partition coefficients of PFOA from the aqueous bulk phase into the phosphatidylcholine bilayers were calculated from the change in $T_{m}$ as a function of PFOA concentration using equation 1 (Table 1). The apparent partition coefficients ranged from $1.0 \times 10^{4}$ to $2.1 \times 10^{4}$ and, thus, were essentially independent of the structure (i.e., chain length of the hydrophobic tails) of the phosphatidylcholines.

For both fluorescent probes, the onset temperature of the phase transition (i.e., the beginning of the transition from the liquid-crystalline to the gel phase of the phosphatidylcholine upon cooling) remained constant for all mixtures over the entire PFOA concentration range studied (Figures 2; see Figures S1, S2 and Wei et al. (2007) for the TMA-DPH results). In contrast, the completion temperature (i.e., the ending of the transition from the liquid-crystalline to the gel phase of the phosphatidylcholine upon cooling) decreased with increasing PFOA concentration, resulting in an increase in the relative width of the phase transition (i.e., the ratio of the transition width of the PFOA-lipid mixture to the transition width of the pure phosphatidylcholine) over the entire concentration range studied. While the concentrationdependent changes in the relative transition width were comparable, especially at low PFOA concentrations (Figure 3), the absolute width was larger for the DMPC system and decreased in the order DMPC > DPPC > DSPC with DPH as fluorescent probe. For example, the transition width of DMPC and DSPC decreased from $14.4 \pm 0.2^{\circ} \mathrm{C}$ (DPH) and $13.8 \pm 0.2^{\circ} \mathrm{C}$ (TMA$\mathrm{DPH})$ to $8.1 \pm 0.2{ }^{\circ} \mathrm{C}(\mathrm{DPH})$ and $7.9 \pm 0.3^{\circ} \mathrm{C}$ (TMA-DPH), respectively, at the highest PFOA concentration investigated $(442 \mu \mathrm{mol} / \mathrm{L})$.

\subsection{Differential scanning calorimetry}

DSC was employed to confirm the changes in the phase behavior of the three PFOAphosphatidylcholine mixtures observed in the fluorescence anisotropy experiments. Overall, the phase behavior of all three mixtures displayed similar features with increasing PFOA concentrations. In short, addition of PFOA resulted in significant peak broadening and a disappearance of the pretransition peak of the three phosphatidylcholines at $X_{D M P C}<0.99$, $X_{D P P C}<0.97$ and $X_{D S P C}<0.94$ (Figure 4 and Figure 5). With increasing mole fraction of PFOA, a shift of the onset (i.e., the beginning of the transition from the gel to the liquidcrystalline phase of the phosphatidylcholine upon heating), maximum and completion temperature (i.e., the ending of the transition from the gel to the liquid-crystalline phase of the phosphatidylcholine upon heating) to lower temperatures was observed. The only exception was the PFOA-DPPC system, where the completion temperature remained approximately constant (Figure 5). Furthermore, the absolute peak width increased with increasing 
concentrations of PFOA in the approximate rank order DMPC > DPPC > DSPC (Figure 4); however, the relative half-width was comparable at mole fractions above 0.8 (Figure 6).

A more complex phase behavior with several phase transitions was observed at low mole fractions of DMPC and DPPC, but not DPSC (Figure 4). In the PFOA-DMPC system, only one main phase transition peak was observed down to a mole fraction of $X_{D M P C}<0.90$, whereas at $X_{D M P C}=0.80$ a maximum transition temperature of $17.0 \pm 0.2^{\circ} \mathrm{C}$ and shoulders at $12.0 \pm$ $0.4{ }^{\circ} \mathrm{C}$ and $18.8 \pm 0.1{ }^{\circ} \mathrm{C}$ were visible. At even lower mole fractions, the phase transition of DMPC became so broad that the onset, maximum and completion temperatures could not be determined. In the PFOA-DPPC system, a shoulder was apparent at $37.8 \pm 0.2{ }^{\circ} \mathrm{C}$ at $X_{D P P C}=$ 0.90 , whereas only one phase transition was apparent in the PFOA-DSPC system over the entire mole fraction range investigated.

\subsection{Dynamic light scattering of PFOA-phosphatidylcholine mixtures}

PFOA was added at different concentrations to aliquots of a common liposome stock solution after extrusion. Therefore, the lipid concentration and initial hydrodynamic diameter was the same for a given PC. In the absence of PFOA, the average hydrodynamic diameters of DMPC, DPPC, and DSPC liposomes at $37^{\circ} \mathrm{C}$ were $159 \pm 3,140 \pm 6$, and $156 \pm 14 \mathrm{~nm}$, respectively (Figure 7A). These results are consistent with liposome extrusion. For DPPC and DSPC, the addition of PFOA did not have a statistically significant effect on vesicle size. However, for DMPC the addition of PFOA increased the average diameter of the vesicles from $159 \pm 3 \mathrm{~nm}$ to $177 \pm 4 \mathrm{~nm}$ in the presence of $442 \mu \mathrm{mol} / \mathrm{L}$ PFOA (PFOA/DMPC mole ratio of 4.42 ). Finally, a reduction in the total normalized scattering intensity, which was defined as the scattering intensity in the presence of PFOA relative to the $0 \mu \mathrm{mol} / \mathrm{L}$ control, was observed for all liposomes with increasing PFOA concentration (Figure 7B). This denotes a less turbid suspension.

\section{Discussion}

\subsection{Steady-state fluorescence anisotropy and DSC experiments}

The present study investigates the effect of PFOA on the phase behavior of phosphatidylcholine model membranes with different degrees of cooperativity of the phase transition (i.e., DMPC, DPPC and DSPC) using DPH and TMA-DPH steady-state fluorescence anisotropy and DSC measurements. We and others have reported a linear decrease in the $T_{m}$ of DPPC with increasing concentrations of PFOA (Inoue et al., 1988a; Xie et al., 2007). Similar to DPPC, the $T_{m}$ of DMPC and DSPC was also significantly altered in the presence of PFOA and decreased linearly with increasing PFOA concentration in both the fluorescence anisotropy measurements and the DSC experiments. The apparent partition coefficients $K_{X}$, which were estimated from the DPH and TMADPH fluorescence anisotropy data using equation 1, ranged from $1.0 \times 10^{4}$ to $2.1 \times 10^{4}$ for all three phosphatidylcholines and, within the experimental error, were essentially independent of the chain length of the phosphatidylcholine. The apparent partition coefficient of PFOA into DPPC model membranes reported by Inoue et al. $\left(K_{X}=0.9\right.$ $\times 10^{4}$ ) is comparable with our results (Inoue et al., 1988a).

In contrast to PFOA, the partition coefficients of related anionic surfactants with an octyl tail, such has sodium octanoate $\left[K_{X}=1.4 \times 10^{2}\right.$ at $\mathrm{pH}=10$ (Inoue et al., 1988a)], potassium octanoate $\left[K_{X}=0.2 \times 10^{2}\right.$ at $\mathrm{pH}=7.4$ (Kamp and Hamilton, 1993) $]$ or sodium octylsulfate $\left[K_{X}=4.7 \times 10^{2}\right.$ (Inoue et al., 1986)], are approximately two orders of magnitude lower compare to the partition coefficient of PFOA. Together, these partition coefficients indicate that PFOA has a high tendency to partition into phosphatidylcholine bilayers, especially compared to analogous short-chain hydrocarbon surfactants (Inoue et al., 1988a; Inoue et al., 1986; Kamp and Hamilton, 1993; Xie et al., 2007). This is a result of the hydrophobic effect, which is more 
pronounced with PFOA due to the hydrophobic character of the perfluorinated tail (i.e., the incorporation of PFOA into the lipid bilayer is energetically more favorable compared to the incorporation of octanoate, the corresponding hydrocarbon compound (Inoue et al., 1988a)). It is important to emphasize that the surfactant is typically added to preformed model membranes in these studies. Therefore, the partition coefficients may only reflect the partitioning of the respective surfactant (i.e., PFOA) into the outer leaflet of the membrane. Further studies are needed to determine if PFOA also partitions into the inner leaflet under the experimental conditions investigated.

Like most other small molecules (Jain and Wu, 1977; Lohner, 1991), PFOA also significantly increased the peak width of the major phase transition of all three phosphatidylcholines in the fluorescence anisotropy and DSC experiments. The absolute peak width of the phosphatidylcholine displayed a clear chain length-dependence (Figure 2 and Figure 5). For example, PFOA more effectively increased the width of the phase transition of DMPC and DPPC compared to DSPC model membranes. Studies of the effect of other lipophilic compounds on the phase behavior of these three phosphatidylcholines, such as tamoxifen derivatives (Custodio et al., 1993a, b) and pyrethroid insecticides (Moya-Quiles et al., 1996a; Moya-Quiles et al., 1996b), have shown a similar rank order. These apparent differences in the phase behavior between the three mixtures reflect the fact that the van der Waals forces between the hydrophobic tails of phospholipids increase with increasing tail length, which increases the cooperativity of the phase transition of the lipid bilayer and, thus, results a smaller transition width in the order DMPC > DPPC > DSPC. However, the change in the relative peak width determined from both the fluorescence and the DSC experiments is comparable for all three PFOA-phosphatidylcholine mixtures and, overall, did not reveal a clear rank order. This observation demonstrates that, similar to the membrane partition coefficients, the relative effect of PFOA on the cooperativity of the phase transition of model membranes is independent of the chain length of the respective phosphatidylcholine.

The effect of PFOA on the $T_{m}$ and the peak width of DMPC, DPPC and DSPC model membranes is characteristic for mixtures of phosphatidylcholines with many hydrocarbon amphiphilic molecules, such as $\mathrm{C}_{5}$ and higher alkanols, alkanoic acids and some nonionic detergents (Eliasz et al., 1976; Jain and Wu, 1977; Lohner, 1991). For example, DSC studies by Eliasz et al. demonstrated that octanoic acid causes a depression of the $T_{m}$ of DPPC, whereas longer chain homologues cause a more moderate decrease (decanoic acid) or even an increase in $T_{m}$ (e.g., alkanoic acids with $\mathrm{C}_{12}$ and longer chains). Similarly, sodium octanoate decreased the $T_{m}$ of lipids isolated from neonatal rat stratum corneum (Cumming and Winfield, 1994). Compared to longer chain alkanoic acids, octanoic acid caused a pronounced increase in the peak width of the phase transition of DPPC, as investigated using DSC (Eliasz et al., 1976).

Analogous to other small molecules, the changes in $T_{m}$ and peak width caused by PFOA indicate a loss of cooperativity of the phase transition. This loss of cooperativity is most likely due to PFOA's preferential partitioning into defects separating cooperative melting units (i.e., highly ordered gel phase clusters), where it is most likely located from approximately $\mathrm{C}_{1}$ through $\mathrm{C}_{8}$ of the methylene region of the phosphatidylcholine acyl chains (Ellena et al., 2002), and an increase in the number of defects due to a net loss of van der Waals interactions and the introduction of free volumes in PFOA-containing bilayers. As a result, the cooperative melting unit in PFOA-containing bilayers is smaller and undergoes a phase transition not only at lower temperatures but also over a broader temperature range.

Although the techniques employed in the present study do not allow us to fully assess the structure of PFOA-containing bilayers, the changes in the pretransition observed in the DSC experiments give additional insights into the effect of PFOA on the molecular organization of phosphatidylcholines at temperatures below the main phase transition. The pretransition is a 
transition from a tilted gel to a ripple gel phase that can be observed for all three phosphatidylcholines in the DSC experiments. Small, hydrophobic molecules can have a drastic effect on the pretransition by causing a change in the tilt angle of the lipid molecules and, thus, are a sensitive measure of changes in the structure of phosphatidylcholine bilayers (Lohner, 1991). Increasing PFOA concentrations caused an increase in the width of the pretransition and, ultimately, abolished the pretransition of all three phosphatidylcholines. Similar changes in the phase behavior of phosphatidylcholines have been reported for other (per-)fluorinated surfactants (Lehmler and Bummer, 2004; Lehmler and Bummer, 2005) and indicate a change in the tilt angle of the phosphatidylcholine molecules in the gel phase. The mole fraction at which PFOA abolished the pretransition depended on the chain length of the phosphatidylcholine and, analogous to PFOA's effect on the peak width, decreased in the order $X_{D M P C}>X_{D P P C}>X_{D S P C}$.

In addition to an effect on the main phase transition, PFOA also altered the fluidity of phosphatidylcholine model membranes. Specifically, PFOA decreased the fluorescence anisotropy values in the gel phase, but increased them in the liquid crystalline phase of all three phosphatidylcholines (Figure 1). The effect of PFOA on the gel phase was very pronounced in the TMA-DPH measurements and suggests an increase in the fluidity of the lipid assemblies present in the gel phase. This observation is in agreement with the effect of PFOA on phosphatidylcholine monolayers at the air-water interface (Matyszewska et al., 2007). In this earlier study, PFOA had a pronounced effect on the transition of phosphatidylcholine monolayers from a liquid-expanded to a liquid-condensed phase at concentrations as low as $10 \mu \mathrm{mol} / \mathrm{L}$. Furthermore, PFOA abolished the phase transition of DMPC and DPPC (subphase temperatures $=13{ }^{\circ} \mathrm{C}$ and $27^{\circ} \mathrm{C}$, respectively) at a concentration of $10 \mu \mathrm{mol} / \mathrm{L}$, resulting in a monolayer in a liquid-expanded state. In the same work, PFOA also reduced the maximum compression modulus of DPPC monolayers from $191 \mathrm{mN} / \mathrm{m}$ to $128 \mathrm{mN} / \mathrm{m}$, which indicates the formation of liquid-like DPPC monolayers (Matyszewska et al., 2007). Together, these studies by Matyszewska and coworkers suggest that PFOA has a fluidizing effect on some phosphatidylcholines assemblies. In the case of phosphatidylcholine bilayers, this effect appears to be more predominant at the location of the TMA-DPH versus the DPH probe (i.e., oriented towards the head group region). In contrast, PFOA has an ordering effect on the liquid crystalline phase in our study, as indicated by an increase in the anisotropy values. Thus, the effect of PFOA on phosphatidylcholine appears to depend, at least to some extent, on the state of the lipid assemblies of interest (e.g., gel versus liquid crystalline phase in bilayers).

One characteristic of hydrocarbon-fluorocarbon mixtures is the highly non-ideal character of these systems. In the case of simple surfactants, this non-ideal character typically results in the formation of hydrocarbon- and fluorocarbon-rich aggregates (Mukerjee and Yang, 1976; Shinoda and Nomura, 1980). However, our fluorescence results (i.e., the linear relationship between $T_{m}$ and PFOA concentration) do not provide any evidence for the formation of PFOArich lipid domains or, possibly, other PFOA/lipid assemblies, such as micelles, with either phosphatidylcholine in the concentration range under investigation $(<0.5 \mathrm{mmol} / \mathrm{L})$. This is in agreement with quasielastic light-scattering experiments demonstrating that PFOA concentrations $\geq 1 \mathrm{mmol} / \mathrm{L}$ are needed to result in "phase separation" or the formation of "aggregates" in DPPC model membranes (Inoue et al., 1988a), but in contrast to our DLS experiments described below which suggest the partial formation of mixed micelles.

Due to the higher PFOA concentrations $(<0.25 \mathrm{~mol} / \mathrm{L})$, multiple phase transitions could be observed in the DSC experiments with the DMPC-PFOA $\left(X_{D M P C} \leq 0.8\right)$ and in the DPPCPFOA $\left(X_{D P P C}=0.9\right)$ systems. Only one main phase transition was observed in the DSPCPFOA system over the entire mole fraction range investigated. These observations indicate that new lipid assemblies were formed at higher phosphatidylcholine and/or PFOA mole 
fractions. These lipid assemblies are likely due to phase separation into hydrocarbon and fluorocarbon-rich domains and/or aggregates.

\subsection{Dynamic Light Scattering}

DLS experiments were performed to assess the effect of PFOA concentration on liposome swelling and solubilization at the biologically relevant PFOA concentrations chosen for this study. The mole fraction of PFOA within the bilayers $x_{S, b}$ can be calculated from the apparent partition coefficients determined by DPH anisotropy (Table 1):

$$
x_{S, b}=K_{X} x_{S}=K_{X}\left(\frac{C_{S}}{C_{S}+C_{L}+55.5}\right)
$$

Equation 2

where $x_{S}$ is the mole fraction of PFOA in water. Values of $x_{S, b}$ of $0.08,0.12$, and 0.16 were calculated at the highest PFOA concentration $\left(C_{S}=442 \mu \mathrm{mol} / \mathrm{L}\right)$ for DMPC, DPPC, and DSPS, respectively. At $37^{\circ} \mathrm{C}$, increasing PFOA concentration yielded a modest increase the hydrodynamic diameter of DMPC liposomes, but did not have a statistically significant effect on DPPC or DSPC (Figure 7A). Based on the fluorescence anisotropy measurements and the corresponding PFOA/lipid ratios, DMPC was in the fluid phase, whereas DPPC and DSPC were in the gel phase during the DLS analysis. Furthermore, fluorescence anisotropy and DSC results indicate that PFOA did reside in both gel and fluid phases. Hence, PFOA was present in all phosphatidylcholine liposomes, but only those with fluid bilayers (DMPC) exhibited swelling. Fluid bilayers can laterally expand more easily than gel bilayers to accommodate surfactant partitioning.

Given the high PFOA/lipid mole ratios (up to 4.42) and the favorable partitioning of PFOA into the bilayers, it was surprising that the addition of PFOA did not have a more profound effect on liposome size. Because mixtures of fluorocarbon and hydrocarbon surfactants can form fluorocarbon and hydrocarbon-rich assemblies (Mukerjee and Yang, 1976), we attribute this to partial liposome solubilization and the formation of mixed PFOA/lipid micelles, which is supported by the scattering intensity results. Scattering intensity is proportional to the particle number density times the diameter to the sixth power $\left(\mathrm{I} \propto \mathrm{ND}^{6}\right)$. Increasing PFOA concentration led to reductions in the normalized scattering intensity for all lipids studied (Figure 7B). Given that PFOA had little effect on liposome diameter, these reductions in scattering intensity can be attributed to a decrease in the number of liposomes present. Based on the Lichtenberg model (Lichtenberg, 1985), which describes turbidity changes when a surfactant is added to a vesicle dispersion, the reduction in scattering intensity at PFOA concentrations $>111 \mu \mathrm{mol} / \mathrm{L}$ ( $\mathrm{PFOA} / \mathrm{lipid}>1.11$ ) is consistent with the onset of micelle formation. In comparison, alkyl sulfate surfactants, such as sodium dodecyl sulfate, are also known to induce mixed micelle formation at high surfactant concentrations (e.g. at SDS/lipid ratios of 2.4) (Silvander et al., 1996). From the partition coefficients $K_{X}$ determined using the DPH fluorescence experiments (Table 1), the concentration of $111 \mu \mathrm{mol} / \mathrm{L}$ corresponds to PFOA mole factions of 0.02, 0.03, and 0.04 in DMPC, DPPC, and DSPC bilayers, respectively. This analysis suggests that the concentration of PFOA needed to induce micelle formation increases with melting temperature or chain length.

Detecting the presence of micelles using DLS in a mixed micelle-liposome solution was not possible because the liposomes dominated the scattering signal. Furthermore, PFOA-rich micelles would be essentially invisible due to the fact that the refractive indices of water (1.33) and PFOA (1.34 (Cotts, 1994; Kissa, 2001)) are nearly identical. Given the low PFOA mole fractions present within liposomes estimated by the apparent partition coefficients, the reduction in scattering intensity is not attributed to a decrease the effective refractive indices of the liposomes. 
With values of $x_{S, b}$ determined using Equation 2, the apparent mole ratio partition coefficient $K_{R}$ can be determined from $K_{X}$ and used to analyze the effects of PFOA partitioning on bilayer curvature strain:

$$
K_{R}=\frac{K_{X}}{55.5}\left(1-x_{S, b}\right)
$$

Equation 3

$K R$ is approximately proportional to the inverse of the surfactant critical micelle concentration (CMC). Surfactant partitioning yields no monolayer curvature strain within a bilayer when the product of $K_{R} \times \mathrm{CMC} \approx 1$, and reduces curvature strain when $>1$. The CMC for PFOA is 25 $\mathrm{mmol} / \mathrm{L}$. Based on $K_{R}$ determined by Equation $3, K_{R} \times \mathrm{CMC}>4$ was calculated for the liposomes. This calculation suggests that PFOA partitioning reduced curvature strain within the bilayers, which could explain why the liposomes were able to accommodate PFOA without significant swelling. It also demonstrates that PFOA favors bilayer partitioning over micelle formation, which is consistent with the preference for charged surfactants to partition into zwitterionic bilayers rather than form micelles where repulsive headgroup interactions are unfavorable (Heerklotz, 2008; Heerklotz and Seelig, 2000). This, coupled with the hydrophobic character of bulky perfluoroalkane tails, may explain the high apparent partition coefficients of PFOA relative to hydrocarbon surfactants and the lack of evidence to support PFOA "phase separation" or "aggregate" formation within the bilayers. However, the presence of phosphatidylcholine lipids within mixed micelles would reduce repulsive headgroup interactions and increase the likelihood of micelle formation. The formation of micelles, in turn, is consistent with the small decrease in scattering intensity at relatively high PFOA/lipid mole ratios.

\section{Conclusion}

The present work is a systematic study of the effect of PFOA on a series of symmetrical, saturated phosphatidylcholines with different degrees of cooperativity of the main phase transition using fluorescence anisotropy, DSC and DLS measurements. PFOA readily partitioned in the bilayers of all three phosphatidylcholines and significantly altered their phase behavior (i.e., linear decrease in $T_{m}$ and increase in peak width), despite significantly different experimental conditions (i.e., PFOA/lipid ratios and PFOA concentrations). The preference for PFOA to partition into bilayers rather than form micelles was further supported by the apparent reduction in lipid monolayer curvature strain. Similar to other lipophilic compounds, the effect of PFOA follow the rank order DMPC > DPPC > DSPC, which suggests that the PFOA-induced effect on the cooperativity of the phase transition decreased with increasing chain length of the phosphatidylcholine and, thus, an increased degree of cooperativity of the phase transition of the pure phosphatidylcholine. This interpretation is further supported by the DLS studies which demonstrated that PFOA can cause swelling of fluid DMPC but not gel phase DPPC and DSPC bilayers, and that the PFOA content within the bilayer at the onset of liposome solubilization (i.e. micelle formation) increased with lipid chain length. However, the relative effect of PFOA on $T_{m}$ and the peak width appeared to be largely independent of the chain-length of the phosphatidylcholine. Overall, the present study raises several important questions that warrant further investigation. Specifically, it will be important to assess PFOAinduced structural changes on a molecular level in mono- and bilayers in both the gel and fluid state, and to investigate the corresponding changes in bilayer morphology in both model bilayers and, ultimately, the lamellar bodies in the lung of PFOA-treated animals. Furthermore, it will be important to further investigate the effect of PFOA on the dynamic function of pulmonary surfactant. 


\section{Supplementary Material}

Refer to Web version on PubMed Central for supplementary material.

\section{Acknowledgments}

The authors thank Dr. Paul Bummer (University of Kentucky) and Dr. Arijit Bose (University of Rhode Island) for access to the DSC and DLS instruments, respectively, and Ashley Cornell and Sean Marnane (University of Rhode Island) for their assistance with the DLS experiments. This work was supported by grants ES05605, ES013661 and ES012475 from the National Institute of Environmental Health Sciences, NIH. Its contents are solely the responsibility of the authors and do not necessarily represent the official views of the funding agencies.

\section{References}

Abbott BD, Wolf CJ, Schmid JE, Das KP, Zehr RD, Helfant L, Nakayama S, Lindstrom AB, Strynar MJ, Lau C. Perfluorooctanoic acid-induced developmental toxicity in the mouse is dependent on expression of peroxisome proliferator-activated receptor-alpha. Toxicol. Sci 2007;98:571-581. [PubMed: 17488742]

Arora M, Bummer PM, Lehmler H-J. Interaction of a partially fluorinated heptadecanoic acid with diacyl phosphatidylcholines of varying chain length. Langmuir 2003;19:8843-8851.

Bernsdorff C, Wolf A, Winter R, Gratton E. Effect of hydrostatic pressure on water penetration and rotational dynamics in phospholipid-cholesterol bilayers. Biophys. J 1997;72:1264-1277. [PubMed: 9138572]

Bothun GD, Knutson BL, Strobel HJ, Nokes SE. Liposome fluidization and melting point depression by pressurized $\mathrm{CO}_{2}$ determined by fluorescence anisotropy. Langmuir 2005;21:530-536. [PubMed: 15641820]

Cotts PM. Solution properties of a group of perfluoropolyethers: Comparison of unperturbed dimensions. Macromolecules 1994;27:6487-6491.

Cumming KI, Winfield AJ. In vitro evaluation of a series of sodium carboxylates as dermal penetration enhancers. Int. J. Pharm 1994;108:141-148.

Custodio JBA, Almeida LM, Madeira VMC. The active metabolite hydroxytamoxifen of the anticancer drug tamoxifen induces structural changes in membranes. Biochim. Biophys. Acta 1993a;1153:308314. [PubMed: 8274502]

Custodio JBA, Almeida LM, Madeira VMC. The anticancer drug tamoxifen induces changes in the physical properties of model and native membranes. Biochim. Biophys. Acta 1993b;1150:123-129. [PubMed: 8347666]

Eliasz AW, Chapman D, Ewing DF. Phospholipid phase transitions. Effects of $n$-alcohols, $n$ monocarboxylic acids, phenylalkyl alcohols and quarternary ammonium compounds. Biochim. Biophys. Acta 1976;448:220-230. [PubMed: 971432]

Ellena JF, Obraztsov VV, Cumbea VL, Woods CM, Cafiso DS. Perfluorooctyl bromide has limited membrane solubility and is located at the bilayer center. Locating small molecules in lipid bilayers through paramagnetic enhancements of NMR relaxation. J. Med. Chem 2002;45:5534-5542. [PubMed: 12459021]

Goerke J. Pulmonary surfactant: functions and molecular composition. Biochim. Biophys. Acta 1998;1408:79-89. [PubMed: 9813251]

Gordon SC, Schurch S, Amrein M, Schoel M. Effects of perfluorinated acids on pulmonary surfactant properties in vitro. Toxicologist 2007;96:91.

Hashizaki K, Taguchi H, Itoh C, Sakai H, Abe M, Saito Y, Ogawa N. Effects of poly(ethylene glycol) (PEG) concentration on the permeability of PEG-grafted liposomes. Chem. Pharm. Bull 2005;53:2731. [PubMed: 15635224]

Heerklotz H. Interactions of surfactants with lipid membranes. Q. Rev. Biophys 2008;41:205-264. [PubMed: 19079805]

Heerklotz H, Seelig J. Correlation of membrane/water partition coefficients of detergents with the critical micelle concentration. Biophys. J 2000;78:2435-2440. [PubMed: 10777739] 
Hu, Wy; Jones, PD.; DeCoen, W.; King, L.; Fraker, P.; Newsted, J.; Giesy, JP. Alterations in cell membrane properties caused by perfluorinated compounds. Comp. Biochem. Physiol. C Toxicol. Pharmacol 2003;135C:77-88. [PubMed: 12781843]

Inoue T, Iwanaga T, Fukushima K, Shimozawa R. Effect of sodium octanoate and sodium perfluorooctanoate on gel-to-liquid-crystalline phase transition of dipalmitoylphosphatidylcholine vesicle membrane. Chem. Phys. Lipids 1988a;46:25-30. [PubMed: 3338099]

Inoue T, Miyakawa K, Shimozawa R. Interaction of surfactants with vesicle membrane of dipalmitoylphosphatidylcholine. Effect on gel-to-liquid-crystalline phase transition of lipid bilayer. Chem. Phys. Lipids 1986;42:261-270. [PubMed: 3829208]

Inoue T, Muraoka Y, Fukushima K, Shimozawa R. Interaction of surfactants with vesicle membrane of dipalmitoylphosphatidylcholine: fluorescence depolarization study. Chem. Phys. Lipids 1988b; 46:107-115. [PubMed: 3342455]

Jain MK, Wu NM. Effect of small molecules on the dipalmitoyl lecithin liposomal bilayer. III. Phase transition in lipid bilayer. J. Membrane Biol 1977;34:157-201.

Kamp F, Hamilton JA. Movement of fatty acids, fatty acid analogs, and bile acids across phospholipid bilayers. Biochemistry 1993;32:11074-11085. [PubMed: 8218171]

Kissa, E. Fluorinated surfactants and repellents (Surfactant Science Series, Vol. 97). New York: Marcel Dekker; 2001.

Koynova R, Caffrey M. Phases and phase transitions of the phosphatidylcholines. Biochim. Biophys. Acta 1998;1376:91-145. [PubMed: 9666088]

Koynova RD, Boyanov AI, Tenchov BG. Gel-state metastability and nature of the azeotropic points in mixtures of saturated phosphatidylcholines and fatty acids. Biochim. Biophys. Acta 1987;903:186196.

Lau C, Anitole K, Hodes C, Lai D, Pfahles-Hutchens A, Seed J. Perfluoroalkyl acids: A review of monitoring and toxicological findings. Toxicol. Sci 2007;99:366-394. [PubMed: 17519394]

Lau C, Thibodeaux Julie R, Hanson Roger G, Narotsky Michael G, Rogers John M, Lindstrom Andrew B, Strynar Mark J. Effects of perfluorooctanoic acid exposure during pregnancy in the mouse. Toxicol. Sci 2006;90:510-518. [PubMed: 16415327]

Lehmler H-J. Synthesis of environmentally relevant fluorinated surfactants-a review. Chemosphere 2005;58:1471-1496. [PubMed: 15694468]

Lehmler H-J, Bummer PM. Mixing of perfluorinated carboxylic acids with dipalmitoylphosphatidylcholine. Biochim. Biophys. Acta 2004;1664:141-149. [PubMed: 15328046]

Lehmler H-J, Bummer PM. Interaction of a partially fluorinated long-chain nicotinate with dipalmitoylphosphatidylcholine. J. Lipid Res 2005;46:2415-2422. [PubMed: 16150829]

Lehmler H-J, Xie W, Bothun GD, Bummer PM, Knutson BL. Mixing of perfluorooctanesulfonic acid (PFOS) potassium salt with dipalmitoyl phosphatidylcholine (DPPC). Colloids Surf. B 2006;51:2529.

Lichtenberg D. Characterization of the solubilization of lipid bilayers by surfactants. Biochim. Biophys. Acta 1985;821:470-478. [PubMed: 4074739]

Lohner K. Effects of small organic molecules on phospholipid phase transitions. Chem. Phys. Lipids 1991;57:341-362. [PubMed: 2054911]

Martin JW, Mabury SA, Solomon KR, Muir DCG. Bioconcentration and tissue distribution of perfluorinated acids in rainbow trout (Oncorhynchus mykiss). Environ. Toxicol. Chem 2003a; 22:196-204. [PubMed: 12503765]

Martin JW, Mabury SA, Solomon KR, Muir DCG. Dietary accumulation of perfluorinated acids in juvenile rainbow trout (Oncorhynchus mykiss). Environ. Toxicol. Chem 2003b;22:189-195. [PubMed: 12503764]

Matyszewska D, Tappura K, Oradd G, Bilewicz R. Influence of perfluorinated compounds on the properties of model lipid membranes. J. Phys. Chem. B 2007;111:9908-9918. [PubMed: 17672485]

Moya-Quiles MR, Munoz-Delgado E, Vidal CJ. Effects of the pyrethroid insecticide permethrin on membrane fluidity. Chem. Phys. Lipids 1996a;79:21-28. [PubMed: 8907239] 
Moya-Quiles MR, Munoz-Delgado E, Vidal CJ. The pyrethroid insecticide deltamethrin modifies the thermotropic properties and lipid packing order of model membranes. Chem. Phys. Lipids 1996b; 83:61-69.

Mukerjee P, Yang AYS. Nonideality of mixing of micelles of fluorocarbon and hydrocarbon surfactants and evidence of partial miscibility from differential conductance data. J. Phys. Chem 1976;80:13881390.

Nakahara H, Nakamura S, Kawasaki H, Shibata O. Properties of two-component Langmuir monolayer of single chain perfluorinated carboxylic acids with dipalmitoylphosphatidylcholine (DPPC). Colloids Surf. B 2005;41:285-298.

Rosen MB, Thibodeaux JR, Wood CR, Zehr RD, Schmid JE, Lau C. Gene expression profiling in the lung and liver of PFOA-exposed mouse fetuses. Toxicology 2007;239:15-33. [PubMed: 17681415]

Shinoda K, Nomura T. Miscibility of fluorocarbon and hydrocarbon surfactants in micelles and liquid mixtures. Basic studies of oil repellent and fire extinguishing agents. J. Phys. Chem 1980;84:365369.

Silvander M, Karlsson G, Edwards K. Vesicle solubilization by alkyl sulfate surfactants: a cryo-TEM study of the vesicle to micelle transition. J. Colloid Interface Sci 1996;179:104-113.

Veldhuizena R, Nagb K, Orgeigc S, Possmayer F. The role of lipids in pulmonary surfactant. Biochim. Biophys. Acta 1998;1408:90-108. [PubMed: 9813256]

Xie W, Kania-Korwel I, Bummer PM, Lehmler H-J. Effect of potassium perfluorooctanesulfonate, perfluorooctanoate and octanesulfonate on the phase transition of dipalmitoylphosphatidylcholine (DPPC) bilayers. Biochim. Biophys. Acta 2007;1768:1299-1308. [PubMed: 17349969]

Zhang X-M, Patel AB, de Graaf RA, Behar KL. Determination of liposomal encapsulation efficiency using proton NMR spectroscopy. Chem. Phys. Lipids 2004;127:113-120. [PubMed: 14706745] 

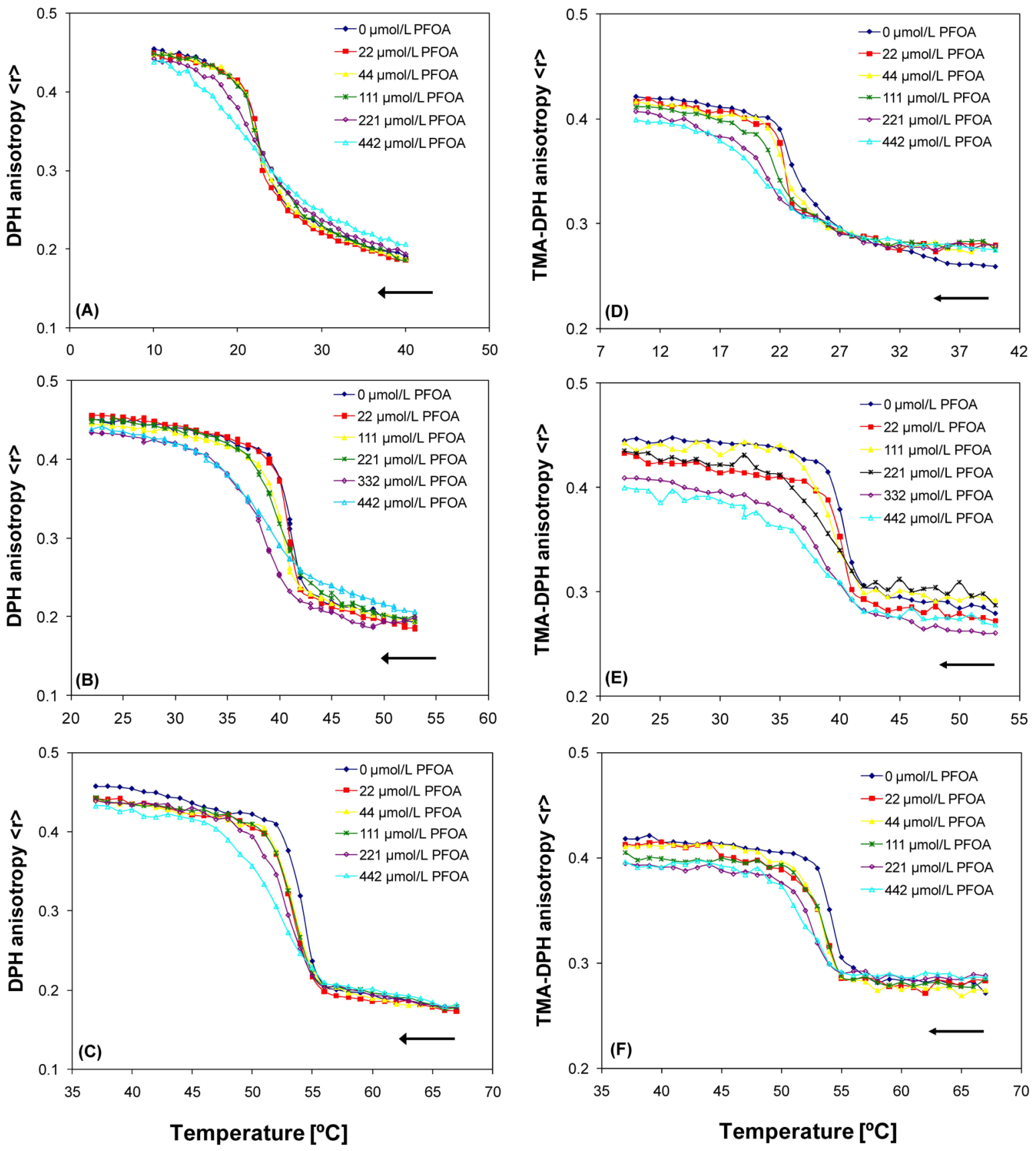

Figure 1.

Temperature dependent changes of phosphatidylcholine-PFOA mixtures obtained from the DPH and TMA-DPH fluorescent anisotropy measurements. DPH measurements: (A) DMPCPFOA, (B) DPPC-PFOA and (C) DSPC-PFOA. TMA-DPH measurements: (D) DMPCPFOA, (E) DPPC-PFOA and (F) DSPC-PFOA. As indicated by the arrows, samples of the respective phosphatidylcholine $(10 \mu \mathrm{mol} / \mathrm{L})$ were cooled at a rate of $0.2 \%$ minute. Each anisotropy-versus-temperature curve is the average of three independent measurements. Figures (B) and (D) are adopted from (Xie et al., 2007) with permission from Elsevier. 

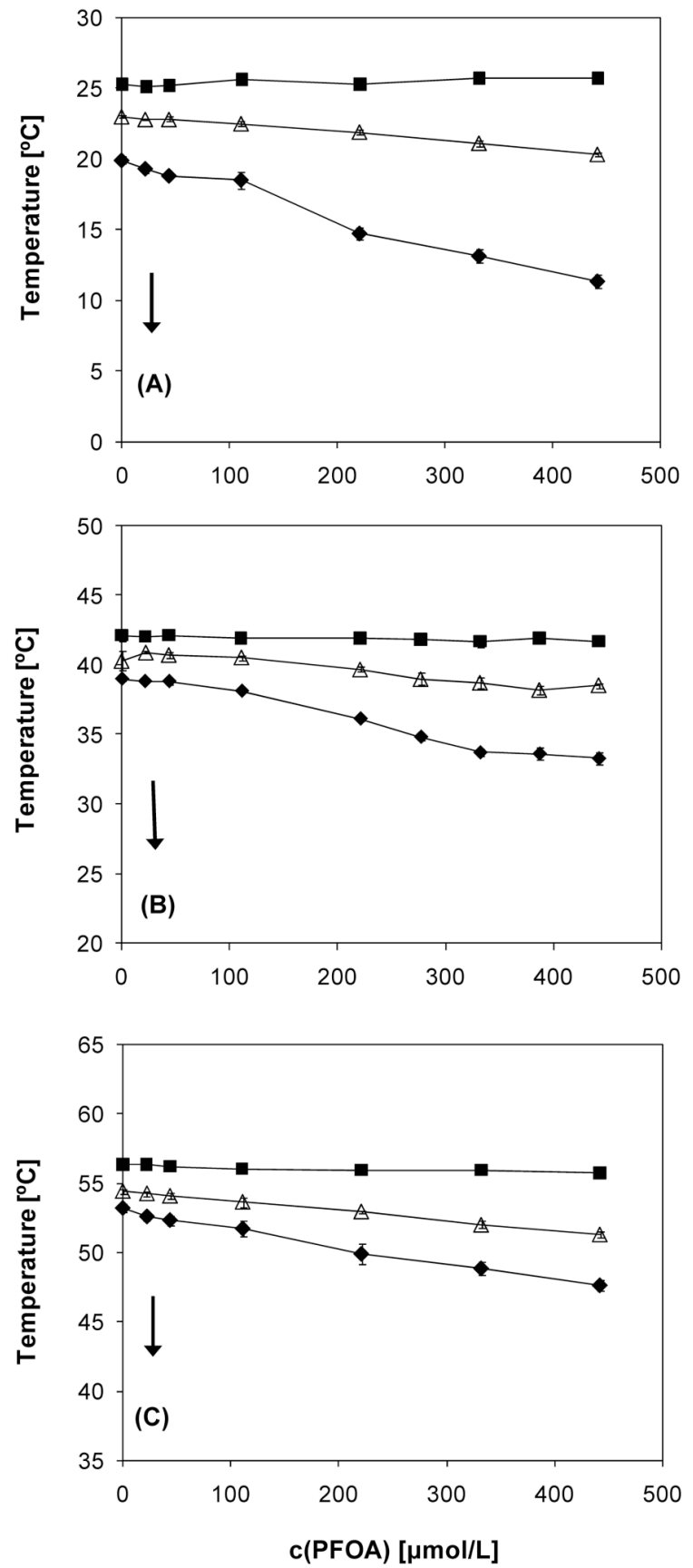

Figure 2.

Partial phase diagram of (A) DMPC-PFOA, (B) DPPC-PFOA (Xie et al., 2007) and (C) DSPCPFOA mixtures obtained from the DPH fluorescent anisotropy measurements. As indicated by the arrows, all samples were cooled at a rate of $0.2 \%$ minute. The data points represent averages of at least three experiments \pm one standard deviation. $\square$ Onset of main phase transition; $\Delta$ maximum of the main phase transition; completion of the main phase transition. 

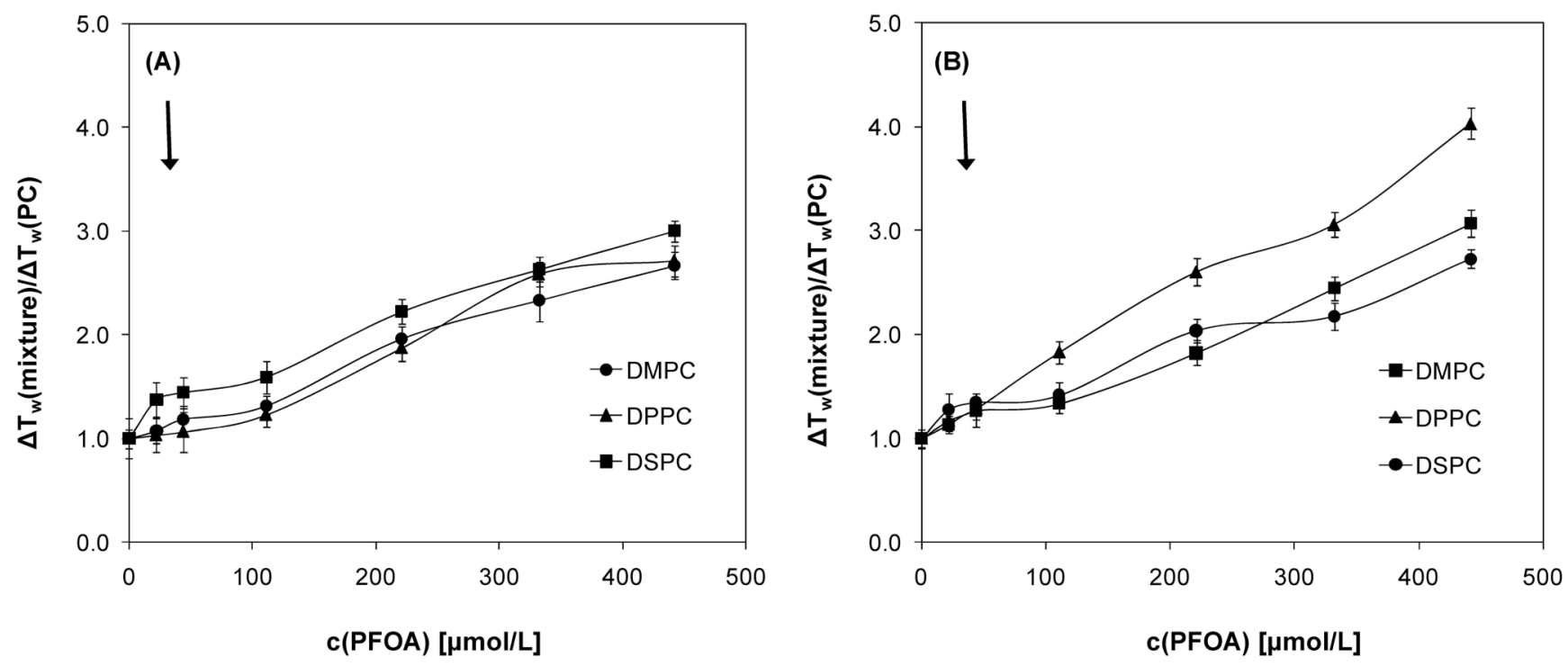

Figure 3.

Concentration-dependence of the relative width of the phase transition of DMPC, DPPC and DSPC mixtures in the presence of 0 to $442 \mu \mathrm{mol} / \mathrm{L}$ PFOA using DPH (A) and TMA-DPH (B) as fluorescent probe. $\Delta \mathrm{T}_{\mathrm{W}}$ (mixture) is the peak width of a phosphatidylcholine-PFOA mixture and $\Delta \mathrm{T}_{\mathrm{W}}(\mathrm{PC})$ is the peak width of the corresponding phosphatidylcholine. All data points are averages of at least three experiments \pm one standard deviation. Samples were cooled at a rate of $0.2 \%$ minute. 


\section{(A) DMPC-PFOA}

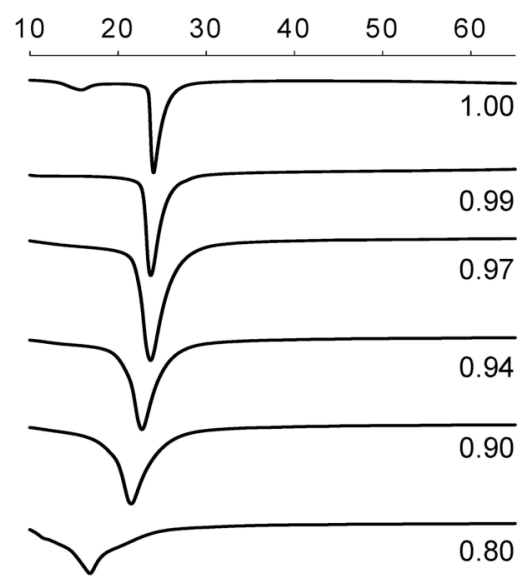

(B) DPPC-PFOA

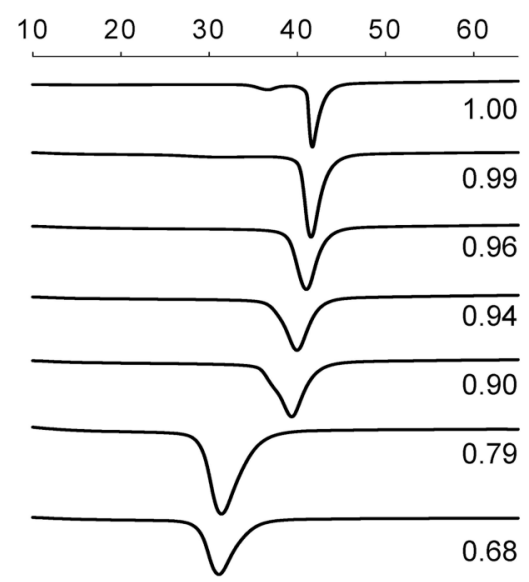

(C) DSPC-PFOA

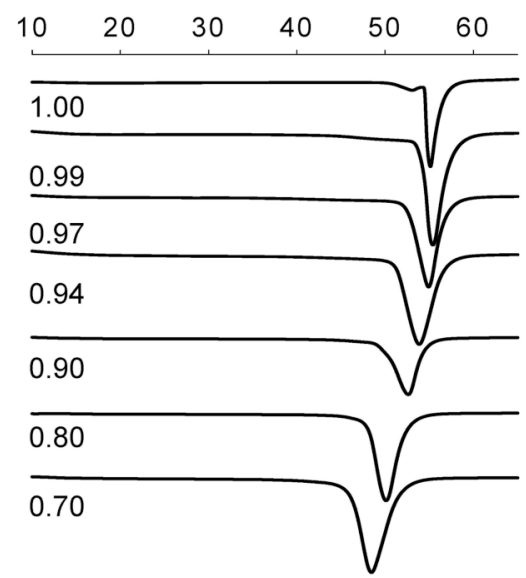

Figure 4.

Typical calorimetric scans for mixtures of (A) DMPC, (B) DPPC (Xie et al., 2007), and (C) DSPC with PFOA in excess water. The mole fraction of the respective phosphatidylcholine is indicated besides each scan. As indicated by the arrows, the heating rate was $5 \%$ minute from $4{ }^{\circ} \mathrm{C}$ to $80^{\circ} \mathrm{C}$ (only the part of the curve with a phase transition is shown). Figure (B) is reprinted from (Xie et al., 2007) with permission from Elsevier. 

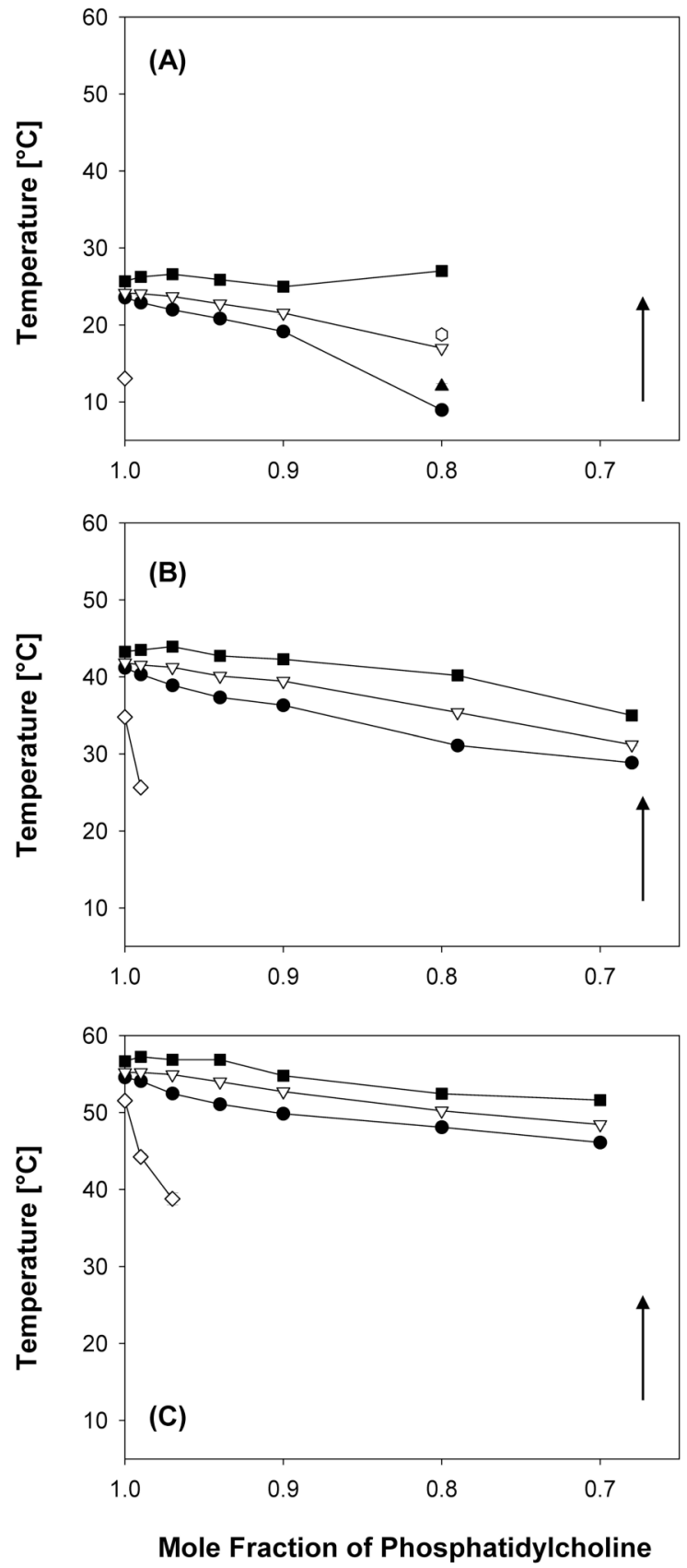

Figure 5.

Partial phase diagram of mixtures of PFOA with (A) DMPC, (B) DPPC and (C) DSPC obtained using differential scanning calorimetry in excess water. All data points are averages of at least three experiments \pm one standard deviation. As indicated by the arrows, the heating rate was $5 \%$ minute from $4{ }^{\circ} \mathrm{C}$ to $80^{\circ} \mathrm{C}$. Completion of main phase transition; $\Delta, \boldsymbol{\Delta}$ and $\square$ maxima of the main phase transition; onset of the main phase transition; $\diamond$ onset of pretransition. Figure (B) is adopted from (Xie et al., 2007) with permission from Elsevier. 


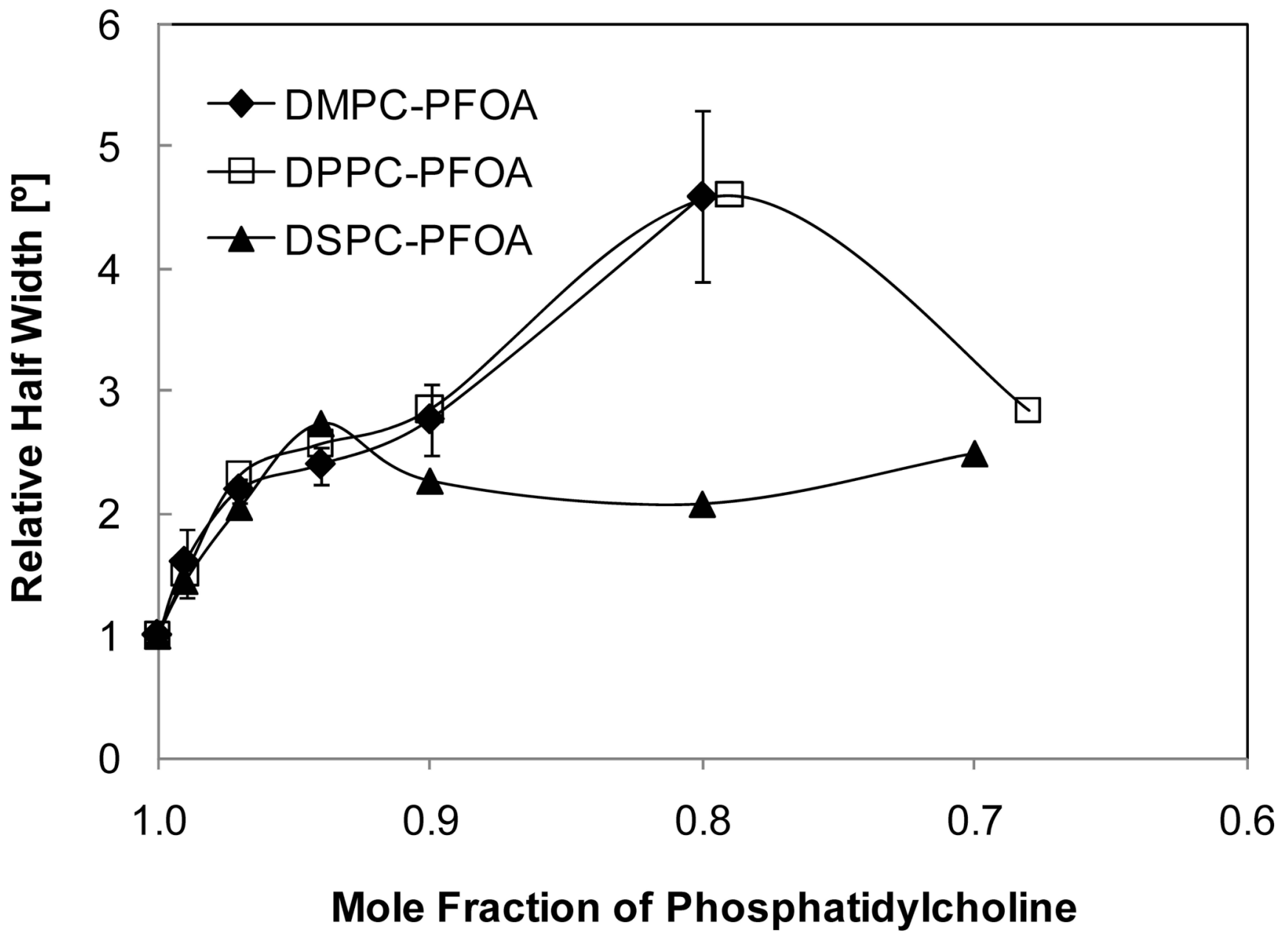

Figure 6.

Half width of main phase transition (relative to half width of the respective pure phosphatidylcholine) of mixtures of PFOA with DMPC, DPPC and DSPC determined with differential scanning calorimetry. All data points are averages of at least three experiments \pm one standard deviation. 

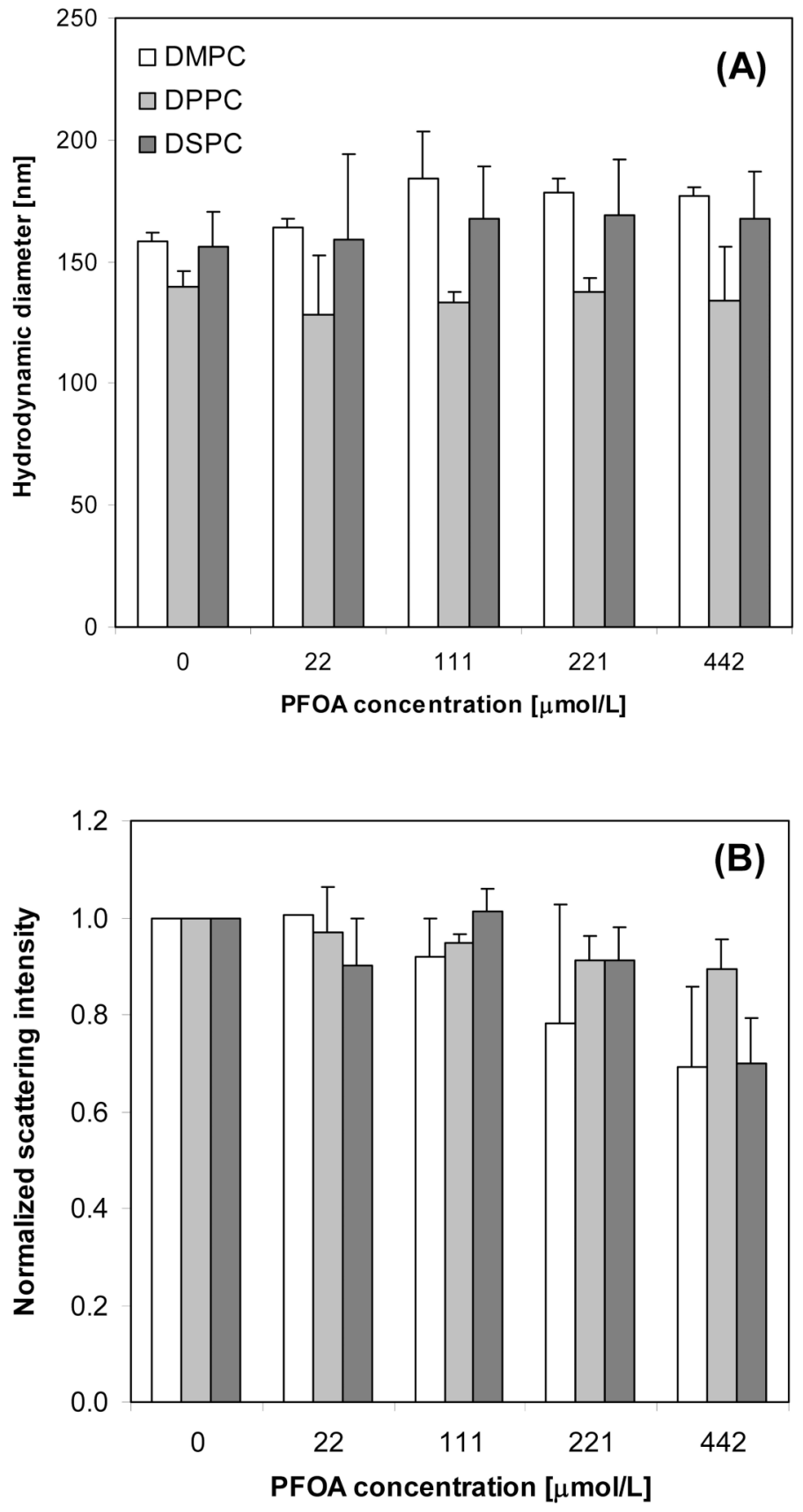

Figure 7.

Average hydrodynamic radius (A) and normalized scattering intensity (B) for DMPC, DPPC, and DSPC $(0.1 \mathrm{mmol} / \mathrm{L}$ lipid $)$ incubated at $37^{\circ} \mathrm{C}$ in the presence of PFOA. Standard deviation bars are shown for duplicate measurements. 
Table 1

Apparent partition apparent partition coefficients of PFOA between phosphatidylcholine model membranes and the bulk aqueous phase.

\begin{tabular}{lll}
\hline Phosphatidylcholine & DPH $\left(\times \mathbf{1 0}^{\mathbf{4}}\right)$ & TMA-DPH $\left(\times \mathbf{1 0}^{\mathbf{4}}\right)$ \\
\hline DMPC & 1.0 & 1.0 \\
DPPC & 1.5 (Lehmler et al., 2006; Xie et al., 2007) & 2.1 (Xie et al., 2007) \\
DSPC & 2.0 & 1.5 \\
\hline
\end{tabular}

